\title{
Actitud hacia las matemáticas de los estudiantes de quinto grado de educación primaria y autoeficacia de los profesores
}

\section{Attitude towards mathematics of fifth grade primary school students and self-efficacy of teachers}

\section{Atitude em relação à matemática de alunos do quinto ano do ensino fundamental e autoeficácia do professor}

\author{
Jaime Segarra ${ }^{1}$, ORCID 0000-0003-4304-2385 \\ Carme Julià2, ORCID 0000-0003-3440-6175 \\ ${ }^{12}$ Universitat Rovira i Virgili, España
}

\begin{abstract}
Resumen: En este trabajo se estudia la actitud hacia las matemáticas de 194 estudiantes de quinto grado. Adicionalmente, se estudia las medias aritméticas entre las creencias de la enseñanza de las matemáticas de los profesores y la actitud de los estudiantes. Se utiliza la Escala de Actitud hacia las Matemáticas (EAM) para medir la actitud de los estudiantes. El Instrumento de Creencias de Eficacia en la Enseñanza de Matemáticas (Mtebi, por su sigla en inglés) se utiliza para medir las creencias de eficacia de los profesores. Los resultados indican que los estudiantes tienen una actitud positiva hacia las matemáticas. El factor confianza tiene puntuaciones más altas. El estudio de las medias indica que los estudiantes con mayor actitud positiva tienen profesores de matemáticas con mayor autoeficacia de la enseñanza de las matemáticas. Los profesores deben considerar a la actitud como un factor importante en el desarrollo cognitivo del estudiante.
\end{abstract}

Palabras clave: actitud; autoeficacia; matemáticas; educación primaria

\begin{abstract}
In this research work, the attitude towards mathematics of 194 fifth grade students is studied. Additionally, the arithmetic mean between teachers' beliefs about mathematics teaching and students' attitudes is studied. The Mathematics Attitude Scale (MAS) is used to measure students' attitudes. The Mathematics Teaching Efficacy Belief Instrument (Mtebi) is used to measure the teachers' efficacy beliefs. The results indicate that students have a favorable level of attitude towards mathematics. The confidence factor has higher scores. The study of means indicates that students with a higher attitude have mathematics teachers with greater teachers' self-efficacy in teaching mathematics. Teachers should consider attitude as an important factor in student cognitive development.
\end{abstract}

Keywords: attitude; self-efficacy; mathematics; primary education

Resumo: Nesta investigação se estuda a atitude em relação à matemática de 194 alunos do quinto ano. Adicionalmente, são estudadas as médias aritméticas entre as crenças do ensino de matemática dos professores e a atitude dos alunos. A Escala de Atitude em relação à Matemática (EAM) é usada para medir a atitude dos alunos. O Instrumento de Crenças de Eficácia do Ensino de Matemática (Mtebi, por sua sigla em inglês) é usado para medir as crenças de eficácia dos professores. Os resultados indicam que os alunos apresentam uma atitude positiva em relação à matemática. $\mathrm{O}$ fator confiança tem pontuações mais altas. O estudo das médias indica que os alunos com atitude mais positiva possuem professores de matemática com maior autoeficácia no ensino de matemática. Os professores devem considerar a atitude como um fator importante no desenvolvimento cognitivo do aluno. 
Palavras-chave: atitude; autoeficácia; matemática; educação primária

Recibido: 18/05/2020

Aceptado: 3/03/2021

Cómo citar:

Segarra, J. \& Julià, C. (2021). Actitud hacia las matemáticas de los estudiantes de quinto grado de educación primaria y autoeficacia de los profesores. Ciencias Psicológicas, 15(1), e2170. doi: https://doi.org/10.22235/cp.v15i1.2170

Correspondencia: Jaime Segarra. Universitat Rovira i Virgili, España. E-mail: jaimerodrigo.segarra@urv.cat

Aprender matemáticas se convirtió en un desafío para la mayoría de los estudiantes. La falta de motivación o la dificultad del aprendizaje hace que muchos estudiantes digan "no soy bueno en matemáticas", antes incluso de intentar resolver problemas de matemáticas. Se considera que este problema general es una cuestión de actitudes hacia las matemáticas más que de falta de capacidades (Colomeischi \& Colomeischi, 2015). También Bausela (2018) indicó que un gran número de escolares experimentan dificultades en las matemáticas.

Algunos investigadores enfatizaron la importancia de estudiar la actitud hacia las matemáticas de los estudiantes (e.g., Aiken, 1970; Auzmendi, 1992; Hourigan \& Leavy, 2019; Ursini \& Sánchez, 2008). Se reconoce que el papel de la motivación y las emociones es crucial para el aprendizaje (Kim, Park \& Cozart, 2014). Cuando los estudiantes carecen de motivación, su proceso de aprendizaje rara vez se inicia y cuando los estudiantes se sienten desesperados, su proceso de aprendizaje se interrumpe fácilmente (Bandura, 1986; Schunk, 1991).

Por otro lado, Aiken (1970) indicó que la actitud es una disposición o tendencia aprendida por parte de un individuo a responder positiva o negativamente a algún objeto, situación, concepto u otra persona. También Neale (1969) definió la actitud hacia las matemáticas como una medida agregada del gusto o desagrado de las matemáticas, una tendencia a participar o evitar actividades matemáticas, la creencia de que uno es bueno o malo en las matemáticas y la creencia de que las matemáticas son útiles o inútiles.

Varios investigadores indicaron que los estudiantes deben tener una actitud positiva hacia las matemáticas, ya que influye en su rendimiento académico en matemáticas (e.g., Bazán, Espinosa, \& Farro, 2001; Figueroa et al., 2012; Salaya, 2006). Sin embargo, algunos investigadores mostraron que los estudiantes de todos los niveles de educación a menudo tienen actitudes negativas hacia las matemáticas (e.g., Boaler, 1997; Michaluk, Stoiko, Stewart \& Stewart, 2018). Sin embargo, es importante que los estudiantes tengan actitudes positivas hacia las matemáticas, ya que las actitudes afectan su desempeño académico y el comportamiento (Cueto, Andrade \& León, 2003).

Por otro lado, en el constructo de creencias, Bandura (1977) indicó que la creencia que tienen las personas son sus propias habilidades para llevar a cabo conductas adecuadas, de manera que les permita alcanzar un objetivo de forma exitosa. La autoeficacia de una persona puede influir en el esfuerzo que realiza en una situación dada y cuánto tiempo persiste en una tarea determinada (Goddard, Hoy \& Woolfolk, 2004). Las expectativas determinan cuánto esfuerzo gastarán las personas y cuánto tiempo persistirán para enfrentar obstáculos y experiencias aversivas. Cuanto más fuerte sea la autoeficacia percibida, más activos serán los esfuerzos.

Teniendo en cuenta la teoría de Bandura $(1977 ; 1997)$, los estudios de las creencias de eficacia de los docentes se han basado en dos dimensiones separadas (Hassan \& Hassan, 2012). La primera dimensión, la autoeficacia de la enseñanza, representa la creencia de un maestro en sus habilidades y capacidades para ser un maestro efectivo. La segunda dimensión, la expectativa de resultados de la enseñanza, es la creencia de un maestro de que la enseñanza efectiva puede lograr un buen aprendizaje 
de los estudiantes, independientemente de factores externos como el entorno familiar, los antecedentes familiares y la influencia de los padres (Swars, Hart, Smith, Smith \& Tolar, 2007).

Varios investigadores han estudiado la actitud hacia las matemáticas de los estudiantes (e.g., Recber, Isiksal \& Koç, 2018; Shahid \& Ullah, 2008). Por otro lado, otros investigadores estudiaron la influencia de las creencias de los profesores en la actitud de los estudiantes (e.g., Chang, 2015).

Nicolaidou y Philippou (2003) estudiaron las relaciones entre las actitudes de los estudiantes hacia las matemáticas, creencias de autoeficacia y la resolución de problemas (logro académico). En una muestra de 238 estudiantes de quinto grado, los autores encontraron que existe una relación significativa entre las actitudes y el logro académico; y una relación más fuerte entre la eficacia y el logro académico. Las actitudes y la eficacia también se correlacionaron y ambos predijeron el logro académico en la resolución de problemas. Sin embargo, la eficacia fue un predictor más poderoso que las actitudes.

Shahid y Ullah (2008) con una muestra de 685 estudiantes de décimo de secundaria aplicaron la escala de actitud matemática de Fennema y Sherman (1976). Los autores indicaron que el éxito de los estudiantes en matemáticas depende de la actitud hacia las matemáticas. También, indicaron que la actitud influye en la tasa de participación en la clase de matemáticas de los alumnos.

Mohamed y Waheed (2011) estudiaron la actitud hacia las matemáticas. A un total de 200 estudiantes de secundaria se les administró un cuestionario para conocer sus actitudes hacia las matemáticas, los estudiantes respondieron preguntas sobre su confianza personal en las matemáticas y la utilidad percibida de las matemáticas. Los autores encontraron que la actitud es un factor que influye en el logro académico de los estudiantes en matemáticas. Adicionalmente, los resultados muestran que los estudiantes tienen una actitud positiva hacia las matemáticas.

Chang (2015) examinó las relaciones entre las creencias de la eficacia de los profesores de quinto de primaria con la autoeficacia y el rendimiento matemático de sus alumnos. Con una muestra de 1244 estudiantes y 58 profesores, los autores encontraron que las creencias de eficacia de los profesores de matemáticas tienen una influencia significativa en la autoeficacia y el logro académico de los alumnos en matemáticas.

La actitud hacia las matemáticas es un constructo que juega un papel importante en la educación matemática (Zan \& Di Martino, 2007). Por otro lado, Charalambous, Philippou y Kyriakides (2008) enfatizaron la importancia de estudiar el constructo de las creencias de la eficacia de la enseñanza de las matemáticas ya que está relacionada con el rendimiento académico de los estudiantes. En este contexto, esta investigación se centra en el estudio de la actitud hacia las matemáticas de los estudiantes de quinto grado de educación primaria. Adicionalmente, se estudia las medias entre la actitud hacia las matemáticas de los estudiantes de quinto grado y la creencia de la eficacia de la enseñanza de las matemáticas que poseen los profesores de matemáticas. A diferencia de otros estudios, en este trabajo se estudiarán cinco factores de la actitud de la matemática y la las creencias de la eficacia de la enseñanza de las matemáticas.

Para cumplir con el propósito de la investigación, se definen tres preguntas:

1. ¿Cuál es el nivel de la actitud hacia las matemáticas de los estudiantes de quinto grado?

2. ¿Hay diferencias significativas entre los cinco factores de la actitud hacia las matemáticas de los estudiantes de quinto grado?

3. ¿Hay una correspondencia entre las medias altas de las creencias de la eficacia de la enseñanza de las matemáticas y la actitud hacia las matemáticas de los estudiantes de quinto grado? 


\section{Método}

\section{Participantes}

Los participantes de este estudio correspondieron a los estudiantes de quinto grado de 5 escuelas de educación primaria de la ciudad Tarragona, España, en el periodo académico 2019-2020. La participación fue voluntaria y anónima. La muestra de este estudio corresponde a $n=194$. Los estudiantes son de quinto grado (de aproximadamente 9 a 11 años de edad). Por otro lado, se tiene una muestra de los profesores de grado (aula) a la que se aplicó el test, concretamente son 8 profesores (matemáticas) de quinto grado de educación primaria.

\section{Instrumentos}

En esta investigación se usaron dos instrumentos: 1) la Escala de Actitud hacia las Matemáticas (EAM) de Auzmendi (1992), se aplicó a los estudiantes de quinto grado; 2) el Instrumento de Creencia de Eficacia de la Enseñanza de las Matemáticas (Mtebi) para profesores (Enochs, Smith \& Huinker, 2000), se aplicó a los profesores de matemáticas.

La Escala de Actitud hacia las Matemáticas (EAM) permite realizar un análisis exhaustivo de la actitud hacia las matemáticas para estudiantes, recogiendo los factores más significativos para su estudio (Auzmendi, 1992). La EAM consta de 25 ítems en una escala Likert de cinco puntos que mide de uno (totalmente en desacuerdo) a cinco (totalmente de acuerdo). Al igual que en el Mtebi, se eliminó el tercer elemento de la escala Likert, que estaba en la versión original de la EAM. La EAM establece cinco factores: agrado (4, 9, 14 y 24), ansiedad $(2,3,7,8,12,13,17,18$ y 22), motivación $(5,10$, y 25$)$, utilidad $(1,6,15,16,19$ y 21$)$ y confianza $(11,20$ y 23). Diez de los ítems de la EAM tienen puntaje inverso $(2,5,7,10,12,15,16,17,22$ y 25$)$. Las respuestas correspondientes a estos ítems deben invertirse antes de agregarse al puntaje total de la EAM. Para la obtención del resultado parcial para cada factor se suma las puntuaciones obtenidas en los ítems correspondientes.

El Stebi (Mtebi adaptado) tiene dos formas: para profesores en formación y para profesores en servicio (Enochs \& Riggs, 1990). En esta investigación el Mtebi fue adaptado para profesores en servicio, las preguntas se reformularon en tiempo presente. Varios investigadores utilizaron el Mtebi en profesores en servicio (e.g., Liu, Jack \& Chiu, 2007; Segarra \& Julià, 2020; Swars, Daane \& Giesen, 2006; Takunyaci \& Takunyaci, 2014). El Mtebi consta de 21 preguntas en una escala Likert de cinco puntos que mide de uno (totalmente en desacuerdo) a cinco (totalmente de acuerdo). Al igual que en (Liu et al., 2007; Segarra \& Julià, 2020), el tercer elemento de la escala Likert, que estaba en la versión original de Mtebi, se eliminó para alentar a los profesores a indicar un nivel de certeza. El Mtebi se compone de dos subescalas. La primera es la subescala de eficacia de enseñanza de matemáticas personal o autoeficacia de la enseñanza (PMTE), que se compone de 13 ítems (2, 3, 5, $6,8,11,15,16,17,18,19,20$ y 21$)$. La otra subescala es la expectativa de resultados de enseñanza de matemáticas (MTOE), que se compone de 8 elementos $(1,4,7,9,10,12,13$ y 14). Ocho de los ítems del PMTE tienen puntaje inverso $(3,6,8,15,17,18,19$ y 21). Las respuestas correspondientes a estos ítems deben invertirse antes de agregarse al puntaje total de PMTE.

\section{Procedimiento}

Los dos instrumentos Mtebi y EAM fueron entregados a los directores de cada institución educativa. Posteriormente, el director fue el encargado de pasar el test de creencias de la eficacia de la enseñanza de las matemáticas (Mtebi) a los profesores de matemáticas de cada aula, en este test los profesores dispusieron de 20 minutos para contestar las 21 preguntas. El test de la actitud hacia las matemáticas (EAM) fue entregado a cada tutor de aula, para que luego de la respectiva autorización de los representantes legales, se aplique el test. Se dispuso de 40 minutos para contestar las 25 preguntas. Para la aplicación del EAM, se realizó el respectivo acompañamiento a los tutores con el propósito de garantizar el control de la aplicación del instrumento. 


\section{Análisis de los datos}

Esta investigación es considerada un estudio cuantitativo. Para determinar la fiabilidad de los resultados obtenidos, se analizó la consistencia interna de las escalas con la prueba Alfa de Cronbach (Cronbach, 1951). La Tabla 1 muestra los coeficientes de alfa de Cronbach de la EAM y sus cinco factores (agrado, ansiedad, motivación, utilidad y confianza). Además, la Tabla 1 muestra los coeficientes de alfa de Cronbach para el Mtebi y sus dos subescalas (PMTE y MOTE). George y Mallery (2003) proporcionaron las siguientes reglas generales para el alfa de Cronbach $(\alpha>0.9-$ Excelente; $\alpha>0.8$ - Bueno; $\alpha>0.7$ - Aceptable; $\alpha>0.6$ - Cuestionable; $\alpha>0.5$ - Malo; y $\alpha<0.5-$ Inaceptable). En el caso de los factores motivación y confianza el valor del alfa de Cronbach es malo, este hecho es ocasionado porque estos dos factores son menos diferenciados y específicos (Auzmendi, 1992). Además, hay que considerar que ambas escalas están compuestas por tres ítems y que la consistencia interna hallada a través del método de $\alpha$ de Cronbach tiende a ser más baja si el número elemento que consta un instrumento es reducido (Auzmendi, 1992). Es importante remarcar que el alfa de Cronbach para el total de la EAM es bueno y para el total del Mtebi es excelente.

Todos los cálculos de la estadística descriptiva e inferencial se realizaron mediante el lenguaje de programación R. Los gráficos se generaron en R Studio y Microsoft Excel.

Tabla 1.

\begin{tabular}{lc} 
Valores de alfa de Cronbach \\
\hline Escalas/Factores & $\alpha$-Cronbach \\
\hline EAM (Estudiantes) & .89 \\
Agrado & .72 \\
Ansiedad & .81 \\
Motivación & .58 \\
Utilidad & .67 \\
Confianza & .51 \\
\hline Mtebi (Profesores) & .91 \\
PMTE & .85 \\
MTOE & .71 \\
\hline
\end{tabular}

\section{Resultados}

\section{Pregunta 1. ¿Cuál es el nivel de la actitud hacia las matemáticas de los estudiantes de quinto grado?}

Este apartado estudia los resultados de la EAM y cada uno de los cinco factores. Para medir la EAM y sus factores, es fundamental que al asignar los puntajes numéricos a las respuestas se tome en cuenta si la afirmación es en sentido positivo o negativo. La Tabla 2 muestra los criterios utilizados para clasificar estas variables como positivo y negativo. Para realizar los criterios de la Tabla 2 se analizó la referencia de Ursini y Sánchez (2019).

Tabla 2.

Nivel de las variables

\begin{tabular}{ll}
\hline Media aritmética & Nivel \\
\hline $1.00-2.50$ & Negativo \\
$2.51-4.00$ & Positivo \\
\hline
\end{tabular}

Los resultados indican que la media aritmética de la actitud hacia las matemáticas es de 3.07. Considerando los valores de la Tabla 2 se puede afirmar que la actitud hacia las matemáticas del 86 $\%$ estudiantes de quinto grado de educación primaria es positiva. Sin embargo, el $14 \%$ de los participantes tienen una actitud negativa hacia las matemáticas. 
A continuación, se estudia las puntuaciones de la media aritmética de cada uno de los factores de la EAM.

Factor agrado. La Tabla 3 muestra las puntuaciones de las medias aritméticas y desviaciones estándar obtenidas en las cuatro preguntas del factor agrado. En este factor los estudiantes obtienen una puntuación media de $2.64(D E=.77)$. El $54 \%$ de estudiantes tienen agrado positivo hacia las matemáticas. Sin embargo, se debe considerar que un gran porcentaje tienen agrado negativo hacia las matemáticas (46\%). La puntuación de la media aritmética obtenida en este factor es inferior a las puntuaciones de la media aritmética obtenida en la EAM $(2.64<3.07)$. Nótese, que la puntuación más alta es la pregunta 14 con una media de 2.96. Por otro lado, la pregunta 9 obtiene la menor puntuación de la media de 2.22 .

Tabla 3.

\begin{tabular}{llcc}
\multicolumn{2}{l}{ Factor agrado hacia las matemáticas } & \\
\hline $\mathrm{N}^{\mathbf{0}}$ & Ítems & $\mathrm{M}$ & $D E$ \\
\hline 4 & Utilizar las matemáticas es una diversión. & 2.77 & 1.02 \\
9 & Me divierte el hablar con otros de matemáticas. & 2.22 & 1.07 \\
14 & Las matemáticas son agradables y estimulantes para mí. & 2.96 & 1.02 \\
24 & Si tuviera oportunidad me inscribiría en más cursos de & 2.38 & 1.03 \\
& matemáticas de los que son obligatorios. & & \\
\hline
\end{tabular}

Factor ansiedad. La Tabla 4 muestra las puntuaciones de las medias aritméticas y las desviaciones estándar obtenidas a cada pregunta del factor ansiedad. En este factor los estudiantes obtienen una puntuación de la media de $3.08(D E=.63)$. El valor de la puntuación de la media correspondiente al factor de ansiedad es superior a la puntuación de la media aritmética de la actitud hacia las matemáticas $(3.08>3.07)$. En este factor se debe tener en cuenta que la escala está codificada de modo que a mayor puntuación menor ansiedad (Flores \& Auzmendi, 2015). Considerando la media y el tipo de codificación de esta escala, se puede decir que los estudiantes tienen un bajo nivel de ansiedad. Es importante destacar que la pregunta18 tiene menor puntuación con una media aritmética de 2.82; la pregunta 22 tiene mayor puntuación $(M=3.27)$.

Tabla 4.

Factor ansiedad hacia las matemáticas

\begin{tabular}{|c|c|c|c|}
\hline $\mathrm{N}^{\mathrm{o}}$ & Ítems & $\mathrm{M}$ & $D E$ \\
\hline 2 & La asignatura de matemáticas se me da bastante mal. & 3.14 & 0.91 \\
\hline 3 & $\begin{array}{l}\text { Estudiar o trabajar con las matemáticas no me asusta en } \\
\text { absoluto. }\end{array}$ & 3.25 & 0.99 \\
\hline 7 & Las matemáticas es una de las asignaturas que más temo. & 3.30 & 0.96 \\
\hline 8 & $\begin{array}{l}\text { Tengo confianza en mí mismo/a cuando enfrento a un problema } \\
\text { de matemáticas. }\end{array}$ & 3.24 & 0.87 \\
\hline 12 & $\begin{array}{l}\text { Cuando me enfrento a un problema de matemáticas me siento } \\
\text { incapaz de pensar con claridad. }\end{array}$ & 3.09 & 0.96 \\
\hline 13 & $\begin{array}{l}\text { Estoy calmado/a y tranquilo/a cuando me enfrento a un } \\
\text { problema de matemáticas. }\end{array}$ & 2.96 & 0.96 \\
\hline 17 & Trabajar con las matemáticas hace que me sienta nervioso/a. & 3.21 & 1.01 \\
\hline 18 & $\begin{array}{l}\text { No me altero cuando tengo que trabajar en problemas } \\
\text { matemáticas. }\end{array}$ & 2.82 & 1.13 \\
\hline 22 & Las matemáticas hacen que me sienta incómodo/a y nervioso/a. & 3.27 & 0.97 \\
\hline
\end{tabular}


Factor utilidad. La Tabla 5 muestra las puntuaciones de las medias aritméticas correspondientes a cada una de las preguntas del factor utilidad. La puntuación de la media de este factor es de $3.01(D E=.58)$. El valor de la puntuación de la media de este factor es inferior a la puntuación de la media aritmética de la EAM $(3.01<3.07)$. Considerando la Tabla 2 se puede afirmar que los estudiantes respondieron en sentido positivo sobre la utilidad. En este factor la puntuación más alta $(M=3.40)$ se obtuvo en la pregunta 6 y la puntuación más baja $(\mathrm{M}=2.52)$ en la pregunta 19. La Tabla 5 muestra que, en todas las preguntas, los estudiantes dan una respuesta en sentido positivo.

Tabla 5.

Factor utilidad hacia las matemáticas

\begin{tabular}{clcc}
\hline${ }^{\prime}$ & Ítems & $M$ & $D E$ \\
\hline 1 & $\begin{array}{l}\text { Considero las matemáticas como una materia muy necesaria } \\
\text { en mis estudios. }\end{array}$ & 3.53 & 0.79 \\
6 & $\begin{array}{l}\text { Quiero llegar a tener un conocimiento más profundo de las } \\
\text { matemáticas. }\end{array}$ & 3.40 & 0.84 \\
15 & $\begin{array}{l}\text { Espero tener que utilizar poco las matemáticas en mi vida } \\
\text { profesional. }\end{array}$ & 3.16 & 1.02 \\
16 & $\begin{array}{l}\text { Considero que existen otras asignaturas más importantes que } \\
\text { las matemáticas para mi futura profesión. }\end{array}$ & 2.56 \\
19 & $\begin{array}{l}\text { Me gustaría tener una ocupación en la cual tuviera que utilizar } \\
\text { las matemáticas. }\end{array}$ & 2.52 & 1.03 \\
21 & $\begin{array}{l}\text { Para mi futuro profesional las matemáticas es una de las } \\
\text { asignaturas más importantes que tengo que estudiar. }\end{array}$ & 2.91 \\
\hline
\end{tabular}

Factor motivación. La Tabla 6 muestra las puntuaciones de las medias aritmética y las desviaciones estándar de los puntajes dados para las preguntas del factor motivación, con una puntuación de la media de $3.18(D E=0.70)$. La puntuación de la media aritmética de este factor es superior a la puntuación de la media aritmética de la EAM $(3.18>3.07)$. El $79 \%$ de estudiantes respondieron de manera positiva a las preguntas del factor motivación. En este factor, la puntuación más alta de la media aritmética es en la pregunta 25 , seguida de la pregunta 5 , y la pregunta con la puntuación más baja es la 10. La Tabla 6 muestra que, en todas las preguntas, los estudiantes dan una respuesta en sentido positivo.

Tabla 6.

Factor motivación hacia las matemáticas

\begin{tabular}{llcc}
\hline $\mathrm{N}^{\mathbf{0}}$ & Ítems & $\mathrm{M}$ & $\mathrm{DE}$ \\
\hline 5 & $\begin{array}{l}\text { La matemática es demasiado teórica para que pueda servirme } \\
\text { de algo. }\end{array}$ & 3.19 & 1.04 \\
10 & $\begin{array}{l}\text { Las matemáticas pueden ser útiles para el que decida realizar } \\
\text { una carrera de "ciencias" pero no para el resto de los } \\
\text { estudiantes. }\end{array}$ & 3.04 & 1.10 \\
25 & $\begin{array}{l}\text { La materia que se imparte en las clases de matemáticas es muy } \\
\text { poco interesante. }\end{array}$ & 3.23 & 0.99 \\
\hline
\end{tabular}


Factor confianza. La Tabla 7 muestra que los valores de las puntuaciones de las medias aritméticas correspondiente a las preguntas del factor confianza. La puntuación de la media obtenida por los estudiantes en este es de $3.44(D E=.55)$. El valor de la puntuación de la media de este factor es superior a la puntuación de la media aritmética de la EAM $(3.44>3.07)$. Por otro lado, el $79 \%$ de los estudiantes respondieron a las preguntas de este factor en sentido positivo. La pregunta 23 tiene la puntuación mayor, seguido por la pregunta 11. Por último, se tiene la pregunta 20 con una puntuación de la media aritmética más baja. La Tabla 7 muestra que, en las tres preguntas, los estudiantes dan una respuesta en sentido positivo.

Tabla 7.

Factor confianza hacia las matemáticas

\begin{tabular}{llcc}
\hline $\mathrm{N}^{\mathrm{o}}$ & Ítems & $M$ & $D E$ \\
\hline 11 & $\begin{array}{l}\text { Tener buenos conocimientos de matemáticas incrementará } \\
\text { mis posibilidades de trabajo. }\end{array}$ & 3.46 & 0.85 \\
20 & $\begin{array}{l}\text { Me provoca una gran satisfacción el llegar a resolver } \\
\text { problemas matemáticos. }\end{array}$ & 3.41 & 0.91 \\
23 & $\begin{array}{l}\text { Si me lo propusiera creo que llegaría a dominar bien las } \\
\text { matemáticas. }\end{array}$ & 3.49 & 0.87 \\
\hline
\end{tabular}

Pregunta 2. ¿Hay diferencias significativas entre los cinco factores de la actitud hacia las matemáticas de los estudiantes de quinto grado?

Para llevar a cabo el estudio, se calculó el puntaje total de las preguntas de cada factor obtenidos por cada participante (el puntaje total se promedia por el número de ítems calificados por el participante). Para estudiar la distribución de los puntajes, la Figura 1 muestra los puntajes de las medias aritméticas de los cinco factores correspondiente a los estudiantes de quinto grado. Específicamente, el diagrama de caja permite identificar los cuartiles, la mediana (representada por líneas horizontales en regiones más delgadas) y la media (denotada con puntos).

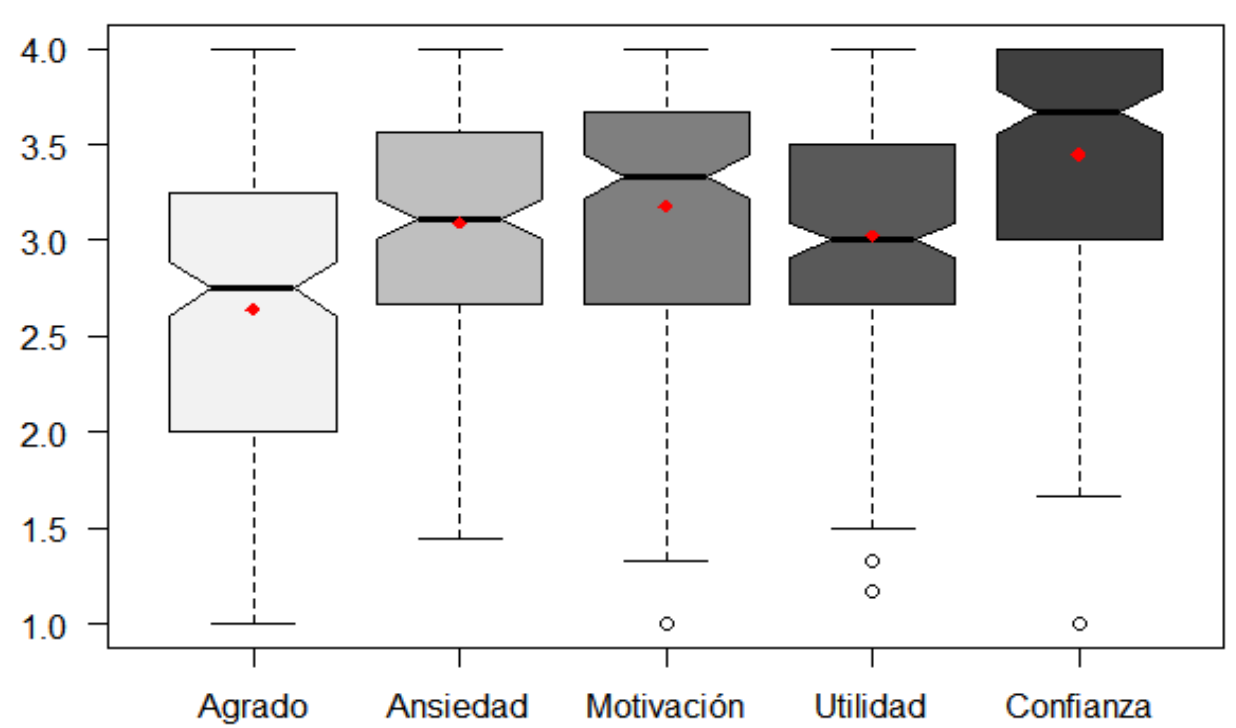

Figura 1. Puntuaciones de las medias de los factores de la actitud hacia las matemáticas. 
La Figura 1 muestra que el rango de valores del factor agrado es claramente más amplio que los otros cuatro grupos. Específicamente, el valor mínimo del factor agrado es el más pequeño (1). En el caso del factor ansiedad, el número de valores pequeños están por debajo de 1.5. En el caso de los factores motivación, utilidad y confianza, hay valores atípicos menores a 1.5. Adicionalmente, se puede apreciar que el factor agrado tiene menor puntuación en la media aritmética.

Asimismo, para verificar si las diferencias entre medias aritméticas son estadísticamente significativas. Se verifica el cumplimiento de las condiciones de hipótesis de normalidad (ShapiroWilk) y homocedasticidad (Bartlett, $p$-valor $>.05$ ). De acuerdo con las condiciones de los datos, se aplica la prueba de Anova. El resultado del Anova indica que hay diferencias significativas entre las medias aritméticas de los cinco grupos $\left(F=41.89, p=1.66 \times 10^{-32}\right)$. Posteriormente, se aplica un análisis post-hoc para estudiar las diferencias significativas entre cada par de los grupos. Específicamente, se aplica la prueba HSD de Tukey (honestly significant difference). La Tabla 8 muestra los $p$-valores, que permiten concluir que la diferencia entre las puntuaciones de las medias aritméticas calculadas es estadísticamente significativa entre la mayoría de pares de los factores de la EAM. Entre los factores pares que no hay diferencias significativas son: ansiedad y motivación ( $p=$ $.68)$; ansiedad y utilidad ( $p=.73)$; y, por último, motivación y utilidad $(p=.079)$.

Tabla 8.

Factor confianza hacia las matemáticas

\begin{tabular}{lcccc}
\hline Factor & Agrado & Ansiedad & Motivación & Utilidad \\
\hline Ansiedad $(\rho)$ & $2.89 \times 10^{-11}$ & & & \\
Motivación $(\rho)$ & $1.23 \times 10^{-13}$ & 0.68 & & \\
Utilidad $(\rho)$ & $7.37 \times 10^{-8}$ & 0.73 & 0.079 & \\
Confianza $(\rho)$ & $1.21 \times 10^{-13}$ & $3.45 \times 10^{-7}$ & $2.36 \times 10^{-4}$ & $1.81 \times 10^{-10}$ \\
\hline
\end{tabular}

Pregunta 3. ¿Hay una correspondencia entre las medias altas de las creencias de la eficacia de la enseñanza de las matemáticas y la actitud hacia las matemáticas de los estudiantes de quinto grado?

La Tabla 9 muestra las medias aritméticas y las desviaciones estándar de las puntuaciones obtenidas por los profesores de quinto de educación primaria. Considerando los valores de la Tabla 2, los profesores dan una afirmación en sentido positivo a las preguntas de la subescala PMTE y MTOE.

Tabla 9.

Media aritmética y desviación estándar (PMTE y MTOE)

\begin{tabular}{lcc}
\hline & $M$ & $D E$ \\
\hline PMTE & 2.97 & 0.35 \\
MTOE & 2.52 & 0.28 \\
\hline
\end{tabular}

La Tabla 10 muestra las medias aritméticas de las puntuaciones obtenidas por los profesores de quinto grado de educación primaria en la autoeficacia de la enseñanza de las matemáticas (PMTE). Además, la Tabla 10 muestra las medias aritméticas de las puntuaciones obtenidos por los estudiantes de quinto grado en la actitud hacia las matemáticas. Específicamente, la Tabla 10 muestra las medias de los ocho grupos estudiados. 
Tabla 10.

Media aritmética del EAM y PMTE

\begin{tabular}{llc}
\hline & $E A M$ & $P M T E$ \\
\hline Grupo 1 & $3.29^{*}$ & $3.38^{*}$ \\
Grupo 2 & $3.27^{*}$ & $3.54^{*}$ \\
Grupo 3 & $3.09^{*}$ & $2.92^{*}$ \\
Grupo 4 & 3.03 & 2.77 \\
Grupo 5 & 2.95 & 2.77 \\
Grupo 6 & 2.93 & 2.38 \\
Grupo 7 & 2.82 & 2.80 \\
Grupo 8 & $3.18^{*}$ & $3.15^{*}$ \\
\hline
\end{tabular}

*Puntuaciones más altas (estadísticamente significativos).

Para determinar qué grupos de estudiantes y profesores obtienen puntuaciones diferentes en la actitud y autoeficacia, respectivamente, se aplica el test t-Student y Anova. Por otro lado, para determinar qué grupos tienen puntuaciones más altas se aplica la prueba de HSD de Tukey. Por tanto, se puede decir que los profesores del grupo 1, grupo 2, grupo 3 y grupo 8 tienen una media significativamente mayor $(p<.05)$ que los otros grupos en el PMTE. De igual manera, los estudiantes del grupo 1, grupo 2, grupo 3 y grupo 8 tienen una media significativamente mayor $(p<.05)$ que los otros grupos en la actitud hacia las matemáticas. Se puede verificar que los grupos coinciden, los estudiantes con mayor actitud tienen profesores con mayor autoeficacia.

La Tabla 11 muestra las medias aritméticas de las puntuaciones obtenidas por los profesores de matemáticas de quinto grado de educación primaria en la expectativa de resultados (MTOE). Además, la Tabla 11 muestra las medias aritméticas de las puntuaciones obtenidos por los estudiantes de quinto grado en la actitud hacia las matemáticas.

Tabla 11.

Media aritmética del EAM y MTOE

\begin{tabular}{llc}
\hline & $E A M$ & $M T O E$ \\
\hline Grupo 1 & $3.29^{*}$ & 2.60 \\
Grupo 2 & $3.27^{*}$ & 2.63 \\
Grupo 3 & $3.09^{*}$ & $2.75^{*}$ \\
Grupo 4 & 3.03 & 2.25 \\
Grupo 5 & 2.95 & 2.38 \\
Grupo 6 & 2.93 & $2.75^{*}$ \\
Grupo 7 & 2.82 & 2.50 \\
Grupo 8 & $3.18^{*}$ & $2.88^{*}$ \\
\hline
\end{tabular}

*Puntuaciones más altas.

Para determinar qué grupos de estudiantes y profesores obtienen puntuaciones diferentes se aplica el test t-Student y Anova. Además, para determinar qué grupos tienen puntuaciones más altas se aplica la prueba de HSD de Tukey. Por tanto, se puede decir que los profesores del grupo 3, 6 y 8 tienen una media significativamente mayor $(p<.05)$ que los otros grupos en el MTOE. Por otro lado, los estudiantes del grupo 1, 2, 3 y 8 tienen una media significativamente mayor $(p<.05)$ que los otros grupos en la actitud hacia las matemáticas. Se puede verificar que a diferencia en el PMTE los grupos no coinciden. Por lo que no se puede decir que los estudiantes con mayor actitud tienen profesores con mayor expectativa de resultados. 


\section{Discusión}

El propósito de esta investigación fue estudiar la actitud hacia las matemáticas de los estudiantes de quinto grado de educación primaria. Adicionalmente, se estudió la media aritmética entre la actitud hacia las matemáticas de los estudiantes y las creencias de la eficacia de los profesores de matemáticas de quinto grado.

Para responder la primera pregunta de investigación, se estudiaron los puntajes de la media aritmética de cada pregunta de los factores de la EAM para el grupo de participantes. Los resultados indicaron que los estudiantes tienen una actitud positiva hacia las matemáticas. Coincidimos con Tezer y Karasel (2010) quienes determinaron que se debe tener actitudes positivas hacia las matemáticas para poder tener un buen desempeño académico. Por otro lado, analizando cada uno de los factores, los estudiantes tienen un agrado positivo hacia las matemáticas. Los resultados indicaron que en las preguntas del factor agrado obtienen puntajes más bajos que los otros factores. La puntuación más baja es en la pregunta 9 (me divierte el hablar con otros de matemáticas). Es importante que se obtenga un nivel alto en el factor de agrado ya que, las personas que vienen a disfrutar de las matemáticas, aumentan su motivación intrínseca y mejoran su aprendizaje (Ma \& Kishor, 1997). En este estudio se puede decir que los participantes tienen un bajo nivel de ansiedad. La ansiedad es una variable facilitadora del rendimiento académico ya que unos niveles moderados de la misma producirán, en el estudiantado, un estado de alerta o atención que mejorará su rendimiento (García, Martínez \& Inglés, 2013). Se determinó que los estudiantes tienen una motivación positiva hacia las matemáticas. Coincidimos con la investigación de Flores y Auzmendi (2018), los participantes obtienen puntuaciones altas en la motivación. También, en el factor de utilidad se puede decir que los estudiantes en todas las preguntas responden en un sentido positivo. Por último, en el factor de confianza se obtienen puntaciones en sentido positivo. Este factor es importante ya que la desconfianza en el empleo de conceptos y procedimientos matemáticos en la resolución de situaciones matemáticas para la vida, impide que se tenga un buen rendimiento académico (Cardoso et al., 2012).

En la segunda pregunta de investigación se determinó que los estudiantes obtienen puntuaciones más altas en el factor confianza en comparación con los otros factores. Por otro lado, se evidencia que en el factor agrado, los estudiantes obtiene la media más baja. Adicionalmente, se determinó que entre los factores ansiedad, utilidad y motivación no se evidenció diferencias significativas, pero si se pudo determinar que estos factores obtuvieron puntuaciones de la media más altas que en el factor de agrado y puntuaciones de la media más bajas que en el factor de confianza. Es importante señalar que es necesario implementar estrategias metodológicas con el propósito de aumentar el agrado hacia las matemáticas.

En la última pregunta de investigación se determinó que los grupos de estudiantes que obtienen una media aritmética más alta en la actitud hacia las matemáticas tiene docentes de matemáticas con mayor autoeficacia de la enseñanza de las matemáticas. Es decir, la autoeficacia de los profesores influye en la actitud hacia las matemáticas de los estudiantes. Por otro lado, esta relación no se observa en la subescala expectativa de los resultados (MTOE). Además, se debe considerar que la autoeficacia del profesor juega un papel importante en la promoción del logro académico de los estudiantes y su desarrollo de la autoeficacia en el aula, los docentes deben realizar esfuerzos para establecer un ambiente de trabajo positivo y colaborativo y de aprendizaje en el servicio que promueva las matemáticas y la educación (Chang, 2015). Considerando estos aspectos importantes de la autoeficacia de la enseñanza de las matemáticas, varios autores han indicado la importancia de proporcionar mejores programas de preparación de profesores, para desarrollar su autoeficacia (e.g., Incikabi, 2013; Sancar, 2013).

Por tanto, sería de gran utilidad incluir una evaluación de la actitud hacia las matemáticas de los estudiantes al iniciar un periodo académico. Lo ideal sería proporcionar a los estudiantes actividades y experiencias para mejorar los factores en las que se obtienen puntuaciones más bajas. 
Por lo que, las autoridades educativas deben considerar como un aspecto importante la autoeficacia de la enseñanza de los profesores ya que esta subescala influye en la actitud de los estudiantes. Es evidente que una baja autoeficacia del docente puede influenciar negativamente en la actitud de los estudiantes, y este a su vez en el rendimiento académico.

En una futura investigación se estudiará la relación entre la actitud hacia las matemáticas de los estudiantes de quinto grado y el logro académico; la autoeficacia de los profesores y el logro académico de los estudiantes; la autoeficacia de los profesores, la actitud de los estudiantes y el logro académico (estudiar el mejor predictor del logro académico).

\section{Referencias}

Aiken, L. (1970). Attitudes towards mathematics. Review of Educational Research, 40, 551-596. doi: 10.3102/00346543040004551

Auzmendi, E. (1992). Las actitudes hacia la matemática-estadística en las enseñanzas media y universitaria. En Características y medición. España: Mensajero.

Bandura, A. (1977). Self-efficacy: Toward a unifying theory of behavioral change. Psychological review, 84(2), 191-215. doi: 10.1037/0033-295X.84.2.191

Bandura, A. (1986). Social foundations of thought and action: A social cognitivetheory. Englewood Cliffs: Prentice Hall.

Bandura, A. (1997). Self-efficacy: The exercise of control. New York: W. H.

Bausela, E. (2018). PISA 2012: Ansiedad y Bajo Rendimiento en Competencia Matemática. Revista Iberoamericana de Diagnóstico y Evaluación-e Avaliação Psicológica, 1(46), 161-173. doi: 10.21865/RIDEP46.1.12

Bazán, J., Espinosa, G., \& Farro, C. (2001). Rendimiento y actitudes hacia la Matemática en el sistema escolar peruano. Documento de Trabajo No 3, Programa MECEP (Medición de la Calidad Educativa Peruana). Lima: Ministerio de Educación.

Boaler, J. (1997). Setting, social class and survival of the quickest. British educational research journal, 23(5), 575-595. doi: 10.1080/0141192970230503

Cardoso, E., Vanegas, E., \& Cerecedo, M. (2012). Diagnóstico sobre las actitudes hacia las matemáticas del estudiantado que inicia sus estudios en tres posgrados en administración de empresas. Revista Electronica EDUCARE, 16(2), 237-253. doi: 10.15359/ree.16-2.15

Chang, Y. (2015). Examining Relationships among Elementary Mathematics Teachers' Efficacy and Their Students' Mathematics Self-efficacy and Achievement. Eurasia Journal of Mathematics, Science and Technology Education, 11(6), 1307-1320. doi: 10.12973/eurasia.2015.1387a

Charalambous, C., Philippou, G., \& Kyriakides, L. (2008). Tracing the development of preservice teachers' efficacy beliefs in teaching mathematics during fieldwork. Educational Studies in Mathematics, 67(2), 125-142.

Colomeischi, A., \& Colomeischi, T. (2015). The students 'emotional life and their attitude toward mathematics learning. Procedia-Social and Behavioral Sciences, 180, 744-750. doi: 10.1016/j.sbspro.2015.02.192

Cronbach, L. (1951). Coefficient alpha and the internal structure of tests. Psychometrika, 16, 1-16.

Cueto S., Andrade, F. \& León, J. (2003). Las actitudes de los estudiantes peruanos hacia la lectura, la escritura, la matemática y las lenguas indígenas. Documento de Trabajo $\mathrm{N}^{\mathrm{o}}$ 44. Lima: GRADE.

Enochs, L., \& Riggs. I. (1990). Further development of an elementary science teaching efficacy belief instrument: A preservice elementary scale. School Science and Mathematics, 90, 695-706. doi: 10.1111/j.1949-8594.1990.tb12048.x 
Enochs, L., Smith, P., \& Huinker, D. (2000). Establishing factorial validity of the mathematics teaching efficacy beliefs instrument. School Science and Mathematics, 100(4), 194-202. doi: 10.1111/j.1949-8594.2000.tb17256.x

Fennema, E., \& Sherman, J. (1976). Fennema-Sherman mathematics attitudes scales: Instruments designed to measure attitudes toward the learning of mathematics by females and males. Journal for research in Mathematics Education, 7(5), 324-326.

Figueroa, S., Perez, A., Baccelli, S., Prieto, G., Moler, E., \& Argentina, P. (2012). Actitudes hacia la estadística en estudiantes de ingeniería. Premisa, 52, 37-49.

Flores, W., \& Auzmendi, E. (2018). Actitudes hacia las matemáticas en la enseñanza universitaria y su relación con las variables género y etnia. Profesorado, Revista de Currículum y Formación del Profesorado, 22(3), 231-251. doi: 10.30827/profesorado.v22i3.8000

García, J., Martínez, M., \& Inglés, C. (2013). ¿Cómo se relaciona la ansiedad escolar con el rendimiento académico? Revista Iberoamericana de Psicología y Salud, 4(1), 63-76.

George, D., \& Mallery, P. (2003). SPSS for Windows step by step: A simple guide and reference. 11.0 update $\left(4^{\circ}\right.$ ed.). Boston: Allyn \& Bacon.

Goddard, R., Hoy, W., \& Woolfolk, A. (2004). Collective efficacy beliefs: Theory developments, empirical evidence, and future directions. Educational researcher, 33(3), 3-13. doi: 10.3102/0013189X033003003

Hassan, A. \& Hassan, T. (2012). Science teaching self-efficacy and outcome expectancy beliefs of secondary school teachers in UAE. International Journal for Research in Education, 32, 122.

Hourigan, M., \& Leavy, A. M. (2019). The influence of entry route to teaching on Irish pre-service primary teachers' attitudes towards mathematics. Journal of Further and Higher Education, 43(7), 869-883. doi: 10.1080/0309877X.2017.1420148

Incikabi, L. (2013). Teacher candidates'efficacy beliefs in mathematics: Play-generated curriculum instruction. Eurasia Journal of Mathematics, Science, \& Technology Education, 9(2), $167-$ 176. doi: 10.12973/eurasia.2013.927a

Kim, C., Park, S. W., \& Cozart, J. (2014). Affective and motivational factors of learning in online mathematics courses. British Journal of Educational Technology, 45(1), 171-185. doi: 10.1111/j.1467-8535.2012.01382.x

Liu, C., Jack, B., \& Chiu, H. (2007). Taiwan elementary teachers' views of science teaching selfefficacy and outcome expectations. International Journal of Science and Mathematics Education, 6(1), 19-35. doi: 10.1007/s10763-006-9065-4

Ma, X., \& Kishor, N. (1997). Assessing the Relationship Between Attitude Toward Mathematics and Achievement in Mathematics: A Meta-Analysis. Journal for Research in Mathematics Education, 28(1), 26-47. doi: 10.2307/749662

Michaluk, L., Stoiko, R., Stewart, G., \& Stewart, J. (2018). Beliefs and Attitudes about Science and Mathematics in Pre-Service Elementary Teachers, STEM, and Non STEM Majors in Undergraduate Physics Courses. Journal of Science Education and Technology, 27(2), 99113.

Mohamed, L. \& Waheed, H. (2011). Secondary students' attitude towards mathematics in a selected school of Maldives. International Journal of humanities and social science, 1(15), 277-281.

Neale, D. (1969). The role of attitudes in learning mathematics. The Arithmetic Teacher, 16(8), 631640.

Nicolaidou, M., \& Philippou, G. (2003). Attitudes towards mathematics, self-efficacy and achievement in problem solving. En M. A. Mariotti (Ed.), European Research in Mathematics Education III (pp.1-11). Italy: University of Pisa.

Recber, S., Isiksal, M., \& Koç, Y. (2018). Investigating self-efficacy, anxiety, attitudes and mathematics achievement regarding gender and school type. Annals of Psychology, 34(1), 41 51. doi: 10.6018/analesps.34.1.229571 
Salaya, A. (2006). La actitud hacia las matemáticas y el rendimiento académico. Revista Memorias, 7(1), 53-62.

Sancar, H. (2013). Effects of video-supported expertise-based training (XBT) on preservice science teachers'self-efficacy beliefs. Eurasia Journal of Mathematics, Science, \& Technology Education, 9(2), 131-141. doi: 10.12973/eurasia.2013.924a

Schunk, D. H. (1991). Self-efficacy and academic motivation. Educational psychologist, 26(3-4), 207-231. doi: 10.1080/00461520.1991.9653133

Segarra, J., \& Julià, C. (2020). Mathematics Teaching Self-Efficacy and Outcome Expectancy of PreService and In-Service Primary Education Teachers. Acta Scientiae, 22(6). doi: 10.17648/acta.scientiae.6049

Shahid, F. \& Ullah, S. (2008). Students' attitude towards mathematics. Pakistan Economic and Social Review, 46(1), 75-83.

Swars, S., Daane, C., \& Giesen, J. (2006). Mathematics anxiety and mathematics teacher efficacy: What is the relationship in elementary preservice teachers? School Science and Mathematics, 106(7), 306-315. doi: 10.1111/j.1949-8594.2006.tb17921.x

Swars, S., Hart, L., Smith, S., Smith, M., \& Tolar, T. (2007). A longitudinal study of elementary preservice teachers' mathematics beliefs and content knowledge. School Science and Mathematics, 107(8), 325-335. doi: 10.1111/j.1949-8594.2007.tb17797.x

Takunyaci, M., \& Takunyaci, M. (2014). Preschool teachers' mathematics teaching efficacy belief. Procedia-Social and Behavioral Sciences, 152, 673-678. doi: 10.1016/j.sbspro.2014.09.261

Tezer, M., \& Karasel, N. (2010). Attitudes of primary school 2nd and 3rd grade students towards mathematics course. Procedia-Social and Behavioral Sciences, 2(2), 5808-5812. doi: 10.1016/j.sbspro.2010.03.947

Ursini, S. \& Sánchez, J. (2019). Actitudes hacia las matemáticas. Qué son. Cómo se miden. Cómo se evalúan. Cómo se modifican. Ciudad de México, México: UNAM, FES Zaragoza.

Ursini, S., \& Sánchez, G. (2008). Gender, technology and attitude towards mathematics: a comparative longitudinal study with Mexican students. ZDM, 40(4), 559-577.

Zan, R., \& Di Martino, P. (2007). Attitude toward mathematics: Overcoming the positive/negative dichotomy. The Montana Mathematics Enthusiast, 3(1), 157-168.

Contribución de los autores: a) Concepción y diseño del trabajo; b) Adquisición de datos; c) Análisis e interpretación de datos; d) Redacción del manuscrito; e) revisión crítica del manuscrito.

J. S. ha contribuido en a, b, c, d, e; y C. J. ha contribuido en a, c, d.

Editora científica responsable: Dra. Cecilia Cracco. 